DOI: 10.12731/2227-930X-2020-1-67-80

УДК 656.13

\title{
ПЕРСПЕКТИВЫ РАЗВИТИЯ АВТОМОБИЛЕЙ С ГИБРИДНОЙ СИЛОВОЙ УСТАНОВКОЙ
}

\author{
Сидоров М.В., Зар Ни Лин, Чижевский К.В., \\ Семенов М.А., Сидоров В.Н.
}

Работа посвящена вопросу развития и компоновке автомобилей с гибридной силовой установкой. В настоящее время наблюдается значительный рост их использования, который обосновывается высокими экономическими и экологическими показателями. Экологические показатели достигаются путем снижением выбросов отработавших газов за счет применения преобразованной и накопленной электрической энергии. Экономические показатели достигаются путем рекуперачии кинетической энергии, тем самым повышия топливную эффективность. При активном развитии автомобилей с гибридной силовой установкой, в том числе на территории России, требуется создание дополнительных условий для обеспечения их технически исправного состояния.

Цель - определение ращчональных схем компоновки гибридной силовой установки.

Метод или методология проведения работы: в статье использованы методы анализа литературных источников.

Результаты: выявлена перспективная компоновка гибридной силовой установки для автомобиля.

Область применения результатов: полученные результатьл целесообразно применять для повышения топливная экономичности автомобилей и снижения токсичности выбросов отработавиих газов.

Ключевые слова: гибридная силовая установка; гибридный автомобиль; электромотор; двигатель внутреннего сгорания; тяговая аккумуляторная батарея; рекуперация; топливная экономич- 
ность; токсичность выбросов отработавших газов; техническое обслуживание; ремонт.

\title{
FUTURE DEVELOPMENT OF CARS WITH HYBRID POWER INSTALLATION
}

\author{
Sidorov M.V., Zar Ni Lin, Chijevsky K.V., \\ Semenov M.A., Sidorov V.N.
}

The work is devoted to the development of cars with hybrid power plant. There is now a significant increase in their use, justified by high economic and environmental indicators. Environmental - reduction of exhaust gas emissions due to use of converted and accumulated electric energy. Economic indicators are achieved by recovery of kinetic energy, thereby increasing fuel efficiency. With the active development of cars with hybrid power plant, including on the territory of Russia, it is necessary to create conditions to ensure their technically serviceable condition.

Objective - determination of rational layout schemes of hybrid power plant.

Method or methodology of work: the article uses methods of analysis of literary sources.

Results: a promising arrangement of hybrid power plant for the car has been revealed.

Field of application: the results obtained are useful for improving the fuel efficiency of automobiles and reducing the toxicity of exhaust gas emissions.

Keywords: hybrid power plant; hybrid car; electric motor; internal combustion engine; traction battery; recuperation; fuel economy; toxicity of exhaust gas emissions; maintenance, repair.

В соответствии со «Стратегией развития автомобильной промышленности Российской Федерации на период до 2025 г.» и «Транспортной стратегии Российской Федерации на период до 2030 года» $[3,4]$ одним из приоритетных направлений инновационного 
развития автомобилестроения в Российской Федерации в соответствии с мировыми тенденциями являются повышение энергоэффективности и экологичности автомобилей, соответствие их конструкции требованиям технических регламентов и других нормативных документов, а также технологии электрификации транспортных средств (электромобили, гибриды). Критическими технологическими операциями при производстве автомобилей являются производство систем управления и диагностики двигателя, накопителей электроэнергии. В сфере развития новых продуктов предполагается применение механизма консорциума в области автомобилестроения, который представляет собой временное объединение органов власти, организаций производителей автомобилей и компонентов, научно-исследовательских и общественных организаций, выполняющих совместные научно-исследовательские, опытно-конструкторские, инженерные и производственные работы, направленные на создание и реализацию высокотехнологичной продукции, включающей, в том числе, развитие гибридных автомобилей [3-8].

Автомобили с гибридной силовой установкой (ГСУ) отличаются от традиционных, наличием двух двигателей (ДВС и электрического). В настоящее время существуют следующие компоновки ГСУ, которые можно разделить на три принципиально отличающиеся схемы $[1,10]$ :

- последовательную (Plug-in Hybrid);

- параллельную (Mild Hybrid);

- последовательно-параллельную (Full Hybrid).

\section{Последовательная схема ГСУ}

Отличительной особенностью последовательной схемы является отсутствие механической связи ДВС и ведущих колёс автомобиля (рис. 1). В этом случае ДВС, работая на постоянном режиме минимального удельного расхода, вращает только ротор электрического генератора, как правило, переменного тока. В приводе ведущих колёс установлен электромотор, который соединен с накопителями электрической энергии. Так как накапливать можно 
только электроэнергию постоянного тока, между ними устанавливается преобразователь (инвертор).

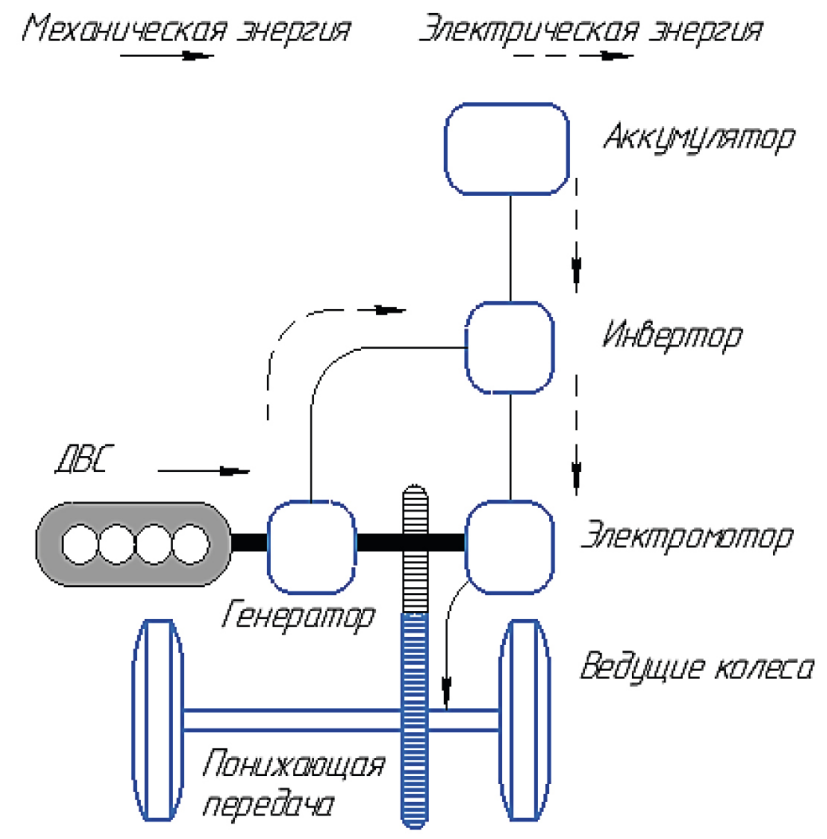

Рис. 1. Последовательная схема КЭУ

В зависимости от режима работы ДВС и условий дорожного движения, электрический ток, вырабатываемый генератором, может подаваться только на электромотор, работающий в этом случае в режиме электродвигателя, или только в накопитель энергии, а может одновременно в оба эти устройства. При замедлении транспортного средства электромотор работает в режиме генератора, обеспечивая рекуперацию кинетической-энергии автомобиля [1].

Достоинствами последовательной схемы являются возможность работы ДВС на режиме минимальных удельных расходов топлива или даже при постоянной частоте вращения коленчатого вала, бесступенчатого изменение передаточного числа трансмиссии и её значительное упрощение. Недостатками являются: малый 
КПД системы передачи энергии от ДВС до ведущих колес; зарядка от аккумуляторных батарей и движение автомобиля происходит только в режиме постоянной работы ДВС.

\section{Параллельная схема ГСУ}

При параллельной схеме ГСУ (рис. 2) ДВС имеет механическую связь с ведущими колёсами. На автомобиле, в отличие от последовательной схемы, установлен только один электромотор. ДВС и электромотор могут работать на трансмиссию автомобиля как раздельно, так и совместно. ДВС через трансмиссию отдаёт энергию ведущим колёсам автомобиля и при избытке его энергии может через электромотор, работающий в этом случае в режиме генератора, питать накопитель [1]. При недостатке энергии ДВС для движения автомобиля дополнительная энергия к ведущим колесам подводится от электромотора, работающего в режиме электродвигателя.

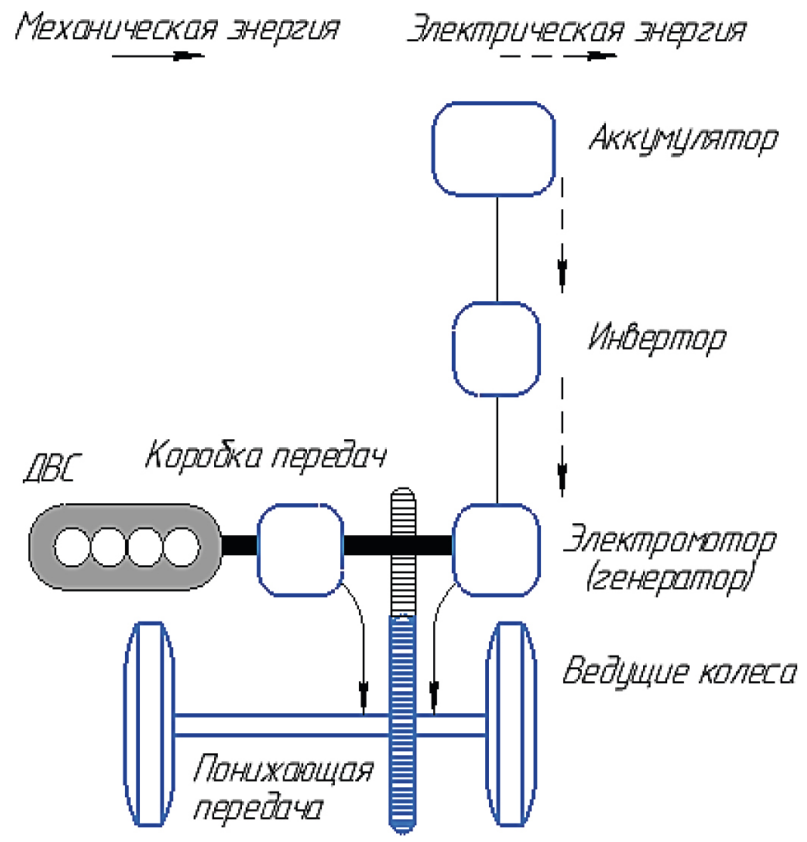

Рис. 2. Параллельная схема ГСУ 
Достоинством параллельной схемы является более высокий КПД передачи энергии от первичного двигателя (ДВС) к ведущим колесам в сравнении с последовательной и возможность применения одной электромашины вместо двух [1]. Недостатками являются обязательное усложнение трансмиссии (так как нужно обеспечить подвод крутящего момента как отдельно от электромотора, так и отдельно от ДВС) и усложнение системы управления трансмиссией и ДВС.

\section{Последовательно-параллельная схема ГСУ}

Последовательно-параллельная схема представляет собой совмещение первых двух [1]. В параллельную схему добавлен дополнительный генератор и делитель мощности (рис. 3). Благодаря этому автомобиль при трогании и на малых скоростях движется только на электрической тяге, ДВС только обеспечивает работу генератора (как при последовательной схеме).

На высоких скоростях крутящий момент на ведущие колеса передается и от двигателя внутреннего сгорания [1]. При повышенных нагрузках (например, при подъеме в гору), когда генератор не в силах обеспечить требуемый ток, электромотор получает дополнительное питание от аккумулятора (параллельная схема).

Поскольку в системе имеется отдельный генератор, заряжающий аккумуляторную батарею, электромотор используется только для привода ведущих колес и во время рекуперативного торможения $[1,9$, 14]. Через планетарный механизм (он же делитель мощности), часть крутящего момента от ДВС частично передается на колеса и частично отбирается для работы генератора, который питает либо электромотор, либо аккумуляторную батарею. Электронный блок управления все время регулирует подачу мощности из обоих источников [1].

Плюсы последовательно-параллельного гибридного двигателя данной схемы, в максимальной топливной экономичности и высокой экологичности. Минусы системы - сложность конструкции и высокая стоимость, поскольку требуется дополнительный генератор, достаточно емкая аккумуляторная батарея и сложный электронный блок управления. 


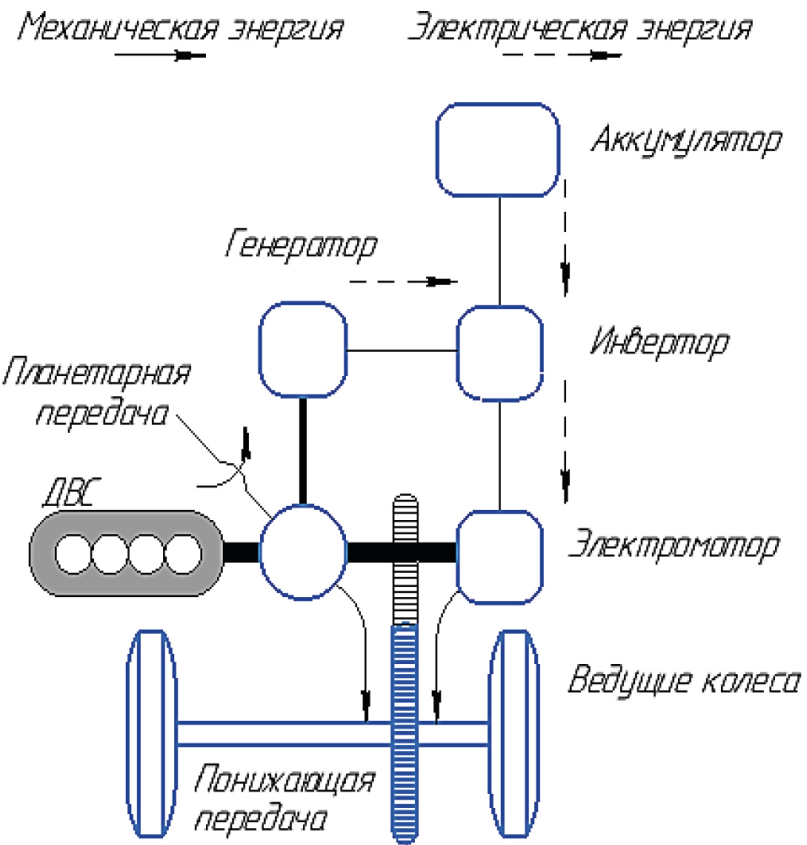

Рис. 3. Последовательно-параллельная схема ГСУ

Автомобили с гибридной силовой установкой имеют следующие основные преимущества [15]:

- экономичность. Расход топлива уменьшается от 20-25\% при интенсивной эксплуатации автомобиля. Двигатель внутреннего сгорания тратит большую энергию при старте автомобиля, так как при движении с места на автомобиль действует большое сопротивление, в это время в работу включается электромотор, питающийся от аккумуляторов, который при увеличении оборотов собственного вала выдает максимальный крутящий момент, благодаря этому бензиновый или дизельный двигатель будут экономить топливо $[15,12,13]$;

- экологичность;

- электродвигатели гибридной силовой установки могут работать как стартер или генератор; 
- может обеспечить больший пробег без дозаправки;

- получение энергии посредством рекуперации, при торможении, а также зарядка аккумуляторных батарей при равномерном движении от электромотора, который работает как генератор.

Недостатки автомобиля с гибридной силовой установкой [11]:

- значительные сложности при утилизации аккумуляторной батареи;

- завышенная стоимость аккумулятора при его ограниченном ресурсе;

- недостаточность соответствующих ремонта, технического обслуживания и диагностирования, их высокая стоимость;

- сложность конструкции.

Тяговая аккумуляторная батарея (ТАБ) является составной частью гибридного автомобиля $[2,5]$. Основными параметрами работы ТАБ являются высокая пиковая и удельная мощность, удельная энергия, высокая степень приспособляемости к зарядке, способность максимально регенерировать энергию торможения, высокий ресурс работы.

В наши дни широко используется свинцово-кислотные батареи. Они относительно недороги и обладают высокой мощностью, надежностью и безопасностью $[2,5]$. Но такие ТАБ имеют низкую удельную энергию, чувствительны к низкой температуре и имеют относительно небольшой ресурс работы, что препятствует их использованию в гибридах. Никель-кадмиевые ТАБ имеют высокую удельную мощность, значительный ресурс работы. Однако они не выдают необходимую энергию для гибридных автомобилей. Никель-водородные батареи имеют более высокий, по сравнению со свинцово- кислотными, ресурс, обладают необходимой энергией и сравнительно безопасны. Такие ТАБ используются в электромобилях и гибридных автомобилях небольшой мощности. К недостаткам никель-водородных батарей относят тепловыделение при высоких температурах, высокую стоимость, саморазряд и необходимость контролировать утечки водорода. Литий-ионные и литий-полимерные 
батареи имеют высокую удельную энергию, мощность, и низкий саморазряд, что вполне обусловливает их применение в гибридных автомобилях. Однако литиевые ТАБ имеют относительно малый ресурс по количеству циклов заряд-разряд и склонность к старению. В настоящее время технология литиевых аккумуляторов активно развивается, в том числе для применения в гибридных автомобилях [1].

Перспективы развития автомобилей с гибридной силовой установкой и их сервисного обслуживания:

- совершенствование конструкции автомобиля;

- модернизация ТАБ;

- приобретение необходимой оснастки автосервиса;

- обеспечение профессионального обучения работников;

- развитие специализации услуг, представляемых предприятиями сервиса автомобилей;

- разработка нормативных и регламентных документов для проведения качественных работ по ремонту, техническому обслуживанию и диагностированию.

Аналитический обзор современного состояния развития автомобилей с гибридной силовой установкой позволил выявить следующие недостатки использования выше изложенных систем: сложность утилизации аккумуляторных батарей; высокая стоимость аккумуляторных батарей, сложность конструкции. Достоинства использования гибридных силовых установок заключается в следующем: экологичность; использование гибридной установки позволяет повысить пробег автомобиля без дозаправки; получение энергии посредством рекуперации.

Тенденция развития и увеличение эксплуатации автомобилей с гибридной силовой установкой обусловлены повышением требований международных стандартов по экологической безопасности и экономичности, ростом цен на углеводородное топливо. Необходим ряд мероприятий, направленных на развитие и создание производственно-технического обеспечения по поддержанию и восстановлению исправного и работоспособного состояния автомобилей с гибридной силовой установкой. Существует потреб- 
ность в разработке технологий выполнения сервисных работ, а также их нормативно-технического обеспечения.

\section{Список литературь}

1. Конструктивные схемы автомобилей с гибридными силовыми установками: учеб. пособие / С.В. Бахмутов, А.Л. Карунин, А.В. Круташов [и др.]. М.: МГТУ «МАМИ», 2007. 71 с.

2. Кузов современного автомобиля: материалы, проектирование и производство: учебное пособие / Пачурин Г.В. [и др.]. Санкт-Петербург: Лань, 2018. 316 c. URL: https://e.lanbook.com/book/107953

3. Распоряжение Правительства РФ от 28 апреля 2018 года N 831-p «Об утверждении Стратегии развития автомобильной промышленности до 2025 года». 1 с.

4. Распоряжение Правительства РФ от 22 ноября 2008 года N 1734-р «Об утверждении Транспортной стратегии Российской Федерации на период до 2030 года (с изменениями на 11 июня 2014 года)». 496 с.

5. Савич, Е.Л. Легковые автомобили: учебник. Минск: Новое знание, 2013. 758 c. URL: https://e.lanbook.com/book/43872

6. Селифонов В.В., Круташов А.В., Баулина Е.Е. Приемно-распределительное устройство преимущественно для комбинированных энергетических установок транспортных средств (варианты) // Патент №55445 на полезную модель. 2006.

7. Селифонов В.В., Круташов А.В., Баулина Е.Е. Электромобиль особо малого класса гибридной энергетической установкой // Автотракторное электрооборудование. 2004. №9. С. 20-22.

8. Умняшкин В.А., Филькин Н.М., Ившин К.С., Скуба Д.В. Автомобили особо малого класса (квадроциклы) с гибридной энергосиловой установкой / Под общ. ред. В.А. Умняшкина. Ижевск: НИЦ «Регуляторная и хаотическая динамика», 2006. 137 с.

9. Умняшкин В.А., Филькин Н.М., Музофаров Р.С., Хамидуллин Р.П. Выбор мощности тягового электродвигателя, двигателя внутреннего сгорания и параметров накопителей гибридных силовых установок автомобилей: Учебное пособие. Ижевск: НИЦ «Регуляторная и хаотическая динамика», 2006. 138 с. 
10. Федотова Ю. Гибридные автомобили: настоящее и будущее // Аймпресс. 2005. №9 (49). С. 23-27.

11. Becerra G., Alvarez-Icaza L., Flores De La Mota I., Mendoza-Soto J.L. Simulation and Optimization Applied to Power Flow in Hybrid Vehicles. // Applied Simulation and Optimization, Springer, 2017, Cham.

12. Hellgren J., Jonasson E. Maximisation of brake energy regeneration in a hybridelec- tric parallel car // Int. J. Electric and Hybrid Vehicles, Vol. 1, No. 1, 2007, pp. 95-121.

13. Kazuaki Shingo, Kaoru Kubo, Toshiaki Katsu, and Yuji Hata. Development of Electric Motors for the TOYOTA Hybrid Vehicle "PRIUS". Toyota Motor Corporation.

14. Kim D-H, Kim J-M, Hwang S-H, and Kim H-S. Optimal brake torque distribution for a four-wheeldrive hybrid electric vehicle stability enhancement // Proc. Instn Mech Engrs, Part D: J. Automobile Engineering, 2007, 221, pp. 1357-1366.

15. Masrur M.A., Garg V.K. Hybrid Electric and Hydraulic Technology Applications in Off-Road Vehicles // Encyclopedia of Sustainability Science and Technology. Springer, New York, 2012.

\section{References}

1. Bahmutov S.V., Karunin A.L., Krutashov A.V. et al. Konstruktivnye shemy avtomobilej s gibridnymi silovymi ustanovkami: ucheb. Posobie [Design diagrams of cars with hybrid power plants]. M.: MGTU «MAMI», 2007. 71 p.

2. Pachurin G.V. et al. Kuzov sovremennogo avtomobilja: materialy, proektirovanie i proizvodstvo [Modern car body: materials, design and production]. Sankt-Peterburg: Lan, 2018. 316 p. https://e.lanbook.com /book/107953/

3. Order of the Government of the Russian Federation of April 28, 2018 N 831-r "On approval of the Strategy for the development of the automotive industry until 2025". $61 \mathrm{p}$.

4. Order of the Government of the Russian Federation of November 22, 2008 N 1734-r "On approval of the Transport Strategy of the Russian Federation for the period until 2030 (as amended on June 11, 2014)". 496 p.

5. Savich E.L. Legkovye avtomobili [Cars]. Minsk: Novoe znanie, 2013. 758 p. https://e.lanbook.com/book/43872. 
6. Selifonov V.V., Krutashov A.V., Baulina E.E. Priemno-raspredelitel'noe ustrojstvo preimushhestvenno dlja kombinirovanny jenergeticheskih ustanovok transportnyh sredstv (varianty) [Receiving and distributing device mainly for combined power plants of vehicles]. Patent №55445, 2006.

7. Selifonov V.V., Krutashov A.V., Baulina E.E. Jelektromobil' osobo malogo klassa gibridnoj jenergeticheskoj ustanovkoj [Electric vehicle of particularly small class hybrid power plant]. Avtotraktornoe jelektrooborudovanie. 2004. №9, pp. 20-22.

8. Umnjashkin V.A., Fil'kin N.M., Ivshin K.S., Skuba D.V. Avtomobili osobo malogo klassa (kvadrocikly) s gibridnoj jenergosilovoj ustanovkoj [Ars of particularly small class (quadrocooks) with hybrid power plant] / ed. V.A. Umnjashkin. Izhevsk, 2006. 137 p.

9. Umnjashkin V.A., Fil'kin N.M., Muzofarov R.S., Hamidullin R.P. Vybor moshhnosti tjagovogo jelektrodvigatelja, dvigatelja vnutrennego sgoranija i parametrov nakopitelej gibridnyh silovyh ustanovok avtomobilej [Selection of power of traction motor, internal combustion engine and parameters of accumulators of hybrid power plants of automobiles]. Izhevsk, 2006. 138 p.

10. Fedotova Ju. Gibridnye avtomobili: nastojashhee i budushhee [Hybrid Cars: Present and Future]. Ajmpress. 2005. №9 (49), pp. 23-27.

11. Becerra G., Alvarez-Icaza L., Flores De La Mota I., Mendoza-Soto J.L. Simulation and Optimization Applied to Power Flow in Hybrid Vehicles. Applied Simulation and Optimization, Springer, 2017, Cham.

12. Hellgren J., Jonasson E. Maximisation of brake energy regeneration in a hybridelec- tric parallel car. Int. J. Electric and Hybrid Vehicles, Vol. 1, No. 1, 2007, pp. 95-121.

13. Kazuaki Shingo, Kaoru Kubo, Toshiaki Katsu, and Yuji Hata. Development of Electric Motors for the TOYOTA Hybrid Vehicle "PRIUS". Toyota Motor Corporation.

14. Kim D-H, Kim J-M, Hwang S-H, and Kim H-S. Optimal brake torque distribution for a four-wheeldrive hybrid electric vehicle stability enhancement. Proc. Instn Mech Engrs, Part D: J. Automobile Engineering, 2007, 221, 1357-1366.

15. Masrur M.A., Garg V.K. Hybrid Electric and Hydraulic Technology Applications in Off-Road Vehicles. Encyclopedia of Sustainability Science and Technology. Springer, New York, 2012. 


\section{ДАННЫЕ ОБ АВТОРАХ}

Сидоров Максим Владимирович, доцент кафедры «Механизация сельскохозяйственного производства», кандидат технических наук

Калужский филиал Российского государственного аграрного университета - МСХА имени К.А. Тимирязева

ул. Вишневского, 27, г. Калуга, Калужская область, 248007, Российская Федерация

sidorov.maxim.79@mail.ru

Зар Ни Лин, аспирант

Калужский филиал Московского государственного технического университета имени Н.Э. Баумана

ул. Баженова, 2, г. Калуга, Калужская область, 248000, Российская Федеращия

zarniznl15@gmail.com

\section{Чижевский Константин Владимирович, студент}

Калужский филиал Московского государственного технического университета имени Н.Э. Баумана

ул. Баженова, 2, г. Калуга, Калужская область, 248000, Российская Федеращия

konstantin201997@yandex.ru

Семенов Максим Андреевич, студент

Калужский филиал Московского государственного технического университета имени Н.Э. Баумана

ул. Баженова, 2, г. Калуга, Калужская область, 248000, Российская Федерачия

traktopuct.maxim@yandex.ru

Сидоров Владимир Николаевич, профессор кафедры «Колесные машины и прикладная механика», доктор технических наук Калужский филиал Московского государственного технического университета имени Н.Э. Баумана 
ул. Баженова, 2, г. Калуга, Калужская область, 248000, Российская Федераџия

\section{DATA ABOUT THE AUTHORS}

Sidorov Maxim Vladimirovich, Associate Professor of the Department of Mechanization of Agricultural Production, Candidate of Technical Sciences, Engineer

Russian Timiryazev State Agrarian University, Kaluzhsky branch 27, Vishnevsky Str., Kaluga, Kaluga region, 248007, Russian Federation

sidorov.maxim.79@mail.ru

ORCID: 0000-0001-8022-866X

Zar Ni Lin, graduate student

Bauman Moscow State Technical University, Kaluga branch 2, Bagenova Str., Kaluga Region, 248000, Russian Federation zarniznl15@gmail.com

Chizhevsky Constantine Vladimirovich, student Bauman Moscow State Technical University, Kaluga branch 2, Bagenova Str., Kaluga Region, 248000, Russian Federation konstantin201997@yandex.ru

Semenov Maxim Andreevich, student

Bauman Moscow State Technical University, Kaluga branch 2, Bagenova Str., Kaluga Region, 248000, Russian Federation traktopuct.maxim@yandex.ru

Sidorov Vlfdimir Nikolaevicsh, Professor of the Department «Wheel Machines and Applied Mechanics», Doctor of Technical Sciences Bauman Moscow State Technical University, Kaluga branch 2, Bagenova Str., Kaluga Region, 248000, Russian Federation sidorov-kaluga@yandex.ru ORCID: 0000-0001-6794-7838 